\title{
Resource Allocation in a Cognitive Radio System with Imperfect Channel State Estimation
}

\author{
Tao Qin, ${ }^{1}$ Cyril Leung, ${ }^{2}$ Chunyan Miao, ${ }^{1}$ and Zhiqi Shen ${ }^{3}$ \\ ${ }^{1}$ School of Computer Engineering, Nanyang Technological University, Singapore 639798 \\ ${ }^{2}$ Department of Electrical and Computer Engineering, The University of British Columbia, Vancouver, B.C., Canada V6T $1 Z 4$
}

${ }^{3}$ School of Electrical and Electronic Engineering, Nanyang Technological University, Singapore 639798

Correspondence should be addressed to Chunyan Miao, ascymiao@ntu.edu.sg

Received 17 October 2009; Accepted 23 February 2010

Academic Editor: Adam Panagos

Copyright (C) 2010 Tao Qin et al. This is an open access article distributed under the Creative Commons Attribution License, which permits unrestricted use, distribution, and reproduction in any medium, provided the original work is properly cited.

\begin{abstract}
Cognitive radio (CR) is a promising concept for improving the utilization of scarce radio spectrum resources. Orthogonal frequency division multiplexing (OFDM) is regarded as a technology which is well matched for CR systems. It is shown that channel estimation errors can result in a severe performance degradation in a multiuser OFDM CR system. A simple back-off scheme is proposed, and simulation results are provided which show that the proposed scheme is very effective in mitigating the negative impact of channel estimation errors.
\end{abstract}

\section{Introduction}

It is believed that the cognitive radio (CR) concept can be used to greatly improve spectral utilization by allowing secondary (unlicensed) users (SUs) to use frequency bands not currently being used by the primary (licensed) users (PUs) in a certain location $[1,2]$. Orthogonal frequency division multiplexing (OFDM) is regarded as a good modulation scheme for CR systems due to its flexibility in allocating resources among SUs [3]. In CR systems, it is important to manage mutual interference problems effectively since primary and secondary users will often simultaneously use adjacent bands.

The problem of power, bit, and subchannel loading for multiuser OFDM CR systems has been studied in [4], in which it is assumed that perfect channel state information is available. In practice, this assumption is often unrealistic, and it is therefore important to study the performance degradation due to imperfect channel state estimation. In [5], the effect of partial channel information in a non-CR multiuser MIMO-OFDM system is discussed. However, the effect of mutual interference which would arise between primary and secondary users in a CR system is not addressed.

A reduced complexity (RC) resource allocation (RA) scheme is proposed for a multiuser OFDM CR system in [4], and it is shown that the scheme provides good performance when perfect channel estimates are available. In this paper, the performance degradation due to imperfect channel state estimation for the RC RA scheme is shown to be quite severe. A simple back-off scheme is proposed and found to be very effective in reducing this degradation.

\section{System Model}

The system model used in this paper is the same as in [4] and is summarized here for the convenience of the reader. We consider the problem of allocating resources on the downlink of an OFDM CR system in which a CR base station (CRBS) serves one primary and $M$ secondary users. The PU band is $W_{p} \mathrm{~Hz}$ wide. On each side of the $\mathrm{PU}$ band, there are $K / 2$ OFDM subchannels, each of width $W_{s} \mathrm{~Hz}$. As the CRBS can transmit simultaneously to the PU and SUs, the PU signal can cause interference to the SUs and vice versa.

The baseband power spectral density (PSD) of the $k$ th subchannel SU signal is modeled as [6]

$$
\Phi_{k}(f)=P_{k} T_{s}\left(\frac{\sin \pi f T_{s}}{\pi f T_{s}}\right)^{2}
$$


where $P_{k}$ is the transmit power of the $k$ th subchannel signal and $T_{s}$ is the symbol duration. The interference power introduced by this signal into the PU band is

$$
I_{k}\left(d_{k}, P_{k}\right)=P_{k} I F_{k}
$$

where

$$
I F_{k}=\int_{d_{k}-W_{p} / 2}^{d_{k}+W_{p} / 2}\left|g_{k}\right|^{2} T_{s}\left(\frac{\sin \pi f T_{s}}{\pi f T_{s}}\right)^{2} d f
$$

is the interference factor for the $k$ th subchannel. In (2) and (3), $g_{k}$ is the channel gain from the CRBS to the PU for the $k$ th subchannel, and $d_{k}$ is the spectral distance between the $k$ th subchannel and the center frequency of the PU band.

The interference power introduced by the PU signal into the $k$ th subchannel band at SU $m$ is

$$
S_{m k}\left(d_{k}\right)=\int_{d_{k}-W_{s} / 2}^{d_{k}+W_{s} / 2}\left|h_{m k}\right|^{2} \Phi_{\mathrm{PU}}(f) d f,
$$

where $h_{m k}$ is the subchannel $k$ gain from the CRBS to SU $m$, and $\Phi_{\mathrm{PU}}(f)$ is the PSD of the signal destined for the PU.

It is assumed that each subchannel can be used for transmission to at most one SU at any given time. Let $P_{m k}$ denote the transmit power allocated to subchannel $k$ of SU $m$. From [7], the maximum number of bits in a symbol transmitted on this subchannel is set to

$$
b_{m k}=\left\lfloor\log _{2}\left(1+\frac{\left|h_{m k}\right|^{2} P_{m k}}{\Gamma\left(N_{0} W_{s}+S_{m k}\right)}\right)\right\rfloor,
$$

where $\lfloor\cdot\rfloor$ denotes the floor function, $N_{0}$ is the one-sided noise PSD, and $S_{m k}$ is given by (4). For convenience, the parameter $\Gamma$ is set to unity in the remainder of this paper.

Let $a_{m k} \in\{0,1\}$ be a subchannel allocation indicator, that is, $a_{m k}=1$ if and only if subchannel $k$ is allocated to SU $m$. Our objective is to maximize the total bit rate for all SUs subject to total transmit power, fairness, and PU interference constraints. Specifically, the optimization problem is expressed as follows:

$$
\max W_{s} \sum_{m=1}^{M} \sum_{k=1}^{K} a_{m k} b_{m k}
$$

subject to

$$
\begin{aligned}
& a_{m k} \in\{0,1\}, \quad \forall m, k \\
& \sum_{m=1}^{M} a_{m k} \leq 1, \quad \forall k, \\
& P_{m k} \geq 0, \quad \forall m, k, \\
& \sum_{m=1}^{M} \sum_{k=1}^{K} a_{m k} P_{m k} \leq P_{\text {total }}, \\
& \sum_{m=1}^{M} \sum_{k=1}^{K} a_{m k} P_{m k} I F_{k} \leq I_{\text {th }},
\end{aligned}
$$

where $P_{\text {total }}$ is the total SU power budget, and $I_{\text {th }}$ is the PU's maximum tolerable interference power. Inequality (8) follows from the assumption that a subchannel can be allocated to at most one SU. Inequalities (10) and (11) correspond to the power and interference constraints, respectively. The nominal bit rate weight (NBRW) for SU $m$ is denoted by $\lambda_{m}$ so that $\lambda_{m} / \sum_{i=1}^{M} \lambda_{i}$ is the fraction of the total number of SU bits loaded that is to be fairly allocated to SU $m$. It is also convenient to denote the total number of bits per symbol period allocated to SU $m$ by $B_{m} \triangleq \sum_{k=1}^{K} b_{m k}$ and define the total bit rate, $R_{m}$, of SU $m$ as $R_{m} \triangleq W_{s} B_{m}$. The total bit rate for all SUs is $R_{s} \triangleq \sum_{m=1}^{M} R_{m}$.

Channel state estimation errors are modeled as follows: let $h$ denote the actual (complex) gain of a channel. This channel could correspond to the $k$ th OFDM subchannel from the CRBS to SU $m$ or to the PU. For simplicity, all channels are assumed to be independently Rayleigh faded, that is, their complex gains are drawn from circularly symmetric, complex Gaussian distributions. The estimated (complex) channel gain is given by

$$
\widehat{h}=h+e
$$

where $e$ is the channel estimation error. For the simulation results presented below, $e$ is assumed to be the outcome of an independent, circularly symmetric, complex Gaussian random variable.

The impact of channel estimation errors on the total SU bit rate, $R_{s}$, can be described as follows. The maximum bit rate, $R_{m k} \triangleq W_{s} b_{m k}$, that can be achieved for SU $m$ on subchannel $k$ depends on the channel gain, $h_{m k}$, the transmit power, $P_{m k}$, and the total interference-plus-noise power, as shown in (5). However, the CRBS knows only $\hat{h}_{m k}$ and not $h_{m k}$. It thus calculates an estimated maximum transmit bit rate, $\hat{R}_{m k}$. If $\hat{R}_{m k}<R_{m k}$, then the opportunity for a higher transmit bit rate is lost. On the other hand, if $\widehat{R}_{m k}>R_{m k}$, then $\hat{R}_{m k}$ exceeds the channel capacity and the actual achieved transmit bit rate is zero. Therefore, the channel estimation errors, if not carefully taken into account in the design of the RA scheme, can result in a severe throughput degradation.

\section{A Scheme for Mitigating Throughput Degradation}

In order to reduce the overall throughput degradation caused by the use of inaccurate channel gain values, we introduce a back-off factor, $B_{G}, 0 \leq B_{G} \leq 1$, such that the RA algorithm uses $B_{G} \times\left|\hat{h}_{m k}\right|^{2}$ instead of $\left|\hat{h}_{m k}\right|^{2}$ as the channel power gain in calculating the estimated maximum transmit bit rate, $\hat{R}_{m k}$. Therefore, (4) and (5) are modified accordingly as

$$
\begin{gathered}
\hat{S}_{m k}\left(d_{k}\right)=B_{G} \int_{d_{k}-W_{s} / 2}^{d_{k}+W_{s} / 2}\left|\hat{h}_{m k}\right|^{2} \Phi_{\mathrm{PU}}(f) d f \\
\hat{b}_{m k}=\left\lfloor\log _{2}\left(1+\frac{B_{G}\left|\hat{h}_{m k}\right|^{2} P_{m k}}{\Gamma\left(N_{0} W_{s}+\hat{S}_{m k}\right)}\right)\right\rfloor .
\end{gathered}
$$


Let $I_{\mathrm{PU}} \triangleq \sum_{k=1}^{K} P_{k} I F_{k}$ be the total interference power introduced into the PU band by SU signals. To control the probability, $P_{o}$, that $I_{\mathrm{PU}}$ exceeds $I_{\mathrm{th}}$, the proposed scheme uses a second back-off factor, $B_{I}$, such that the RA algorithm uses $B_{I} \times I_{\text {th }}$ instead of $I_{\text {th }}$ as the target PU interference power threshold value. A lower value of $P_{o}$ generally requires a lower $B_{I}$ value.

From (14), the incremental power required for transmitting one bit to SU $m$ on subchannel $k$ is given by

$$
\Delta P_{m k}=\frac{N_{0} W_{s}+\hat{S}_{m k}}{B_{G}\left|\hat{h}_{m k}\right|^{2}} 2^{\hat{b}_{m k}} .
$$

From (2) and (15), the incremental interference power generated by such a transmission to the primary user is

$$
\Delta I_{m k}=\Delta P_{m k} I F_{k} .
$$

The MP, MI, and RC RA algorithms were proposed in [4] to improve the throughput in a multiuser OFDM-based CR system. In this paper, modified versions, referred to as $\mathrm{m}$ $\mathrm{MP}, \mathrm{m}-\mathrm{MI}$, and $\mathrm{m}-\mathrm{RC}$, are proposed to mitigate the negative impact of channel estimation errors. Pseudocode listings of these three algorithms are provided below. In the algorithms, $\widehat{B}_{m}$ is the estimated total number of bits allocated to SU $m$ and $P_{\mathrm{SU}}$ is the total transmit power of SUs.

The m-MP algorithm is used to determine the interference power, $I_{\mathrm{MP}}$, introduced into the PU band if, at each bit loading, we choose the subchannel which minimizes the incremental power needed for the selected SU.

\section{Algorithm m-MP}

(1) Step 1-Initialization

(a) Set $P_{\mathrm{SU}}=0, I_{\mathrm{MP}}=0$.

(b) Set $\widehat{B}_{m}=0$ for $m \in\{1,2, \ldots, M\}$.

(c) Set $\hat{b}_{m k}=0$, and calculate $\Delta P_{m k}$ as in (15), for $m \in$ $\{1,2, \ldots, M\}$ and $k \in\{1,2, \ldots, K\}$.

\section{(2) Step 2}

(a) Determine $m^{*}=\arg \min _{m} \widehat{B}_{m} / \lambda_{m}$; ties are first broken in decreasing order of $\lambda$ then randomly.

(b) Determine $k_{P}=\arg \min _{k} \Delta P_{m^{*} k}$.

(c) If $P_{\mathrm{SU}}+\Delta P_{m^{*} k_{p}} \leq P_{\text {total }}$, perform the following updates: $\hat{B}_{m^{*}}=\widehat{B}_{m^{*}}+1, P_{\mathrm{SU}}=P_{\mathrm{SU}}+\Delta P_{m^{*} k_{P}}, \hat{b}_{m^{*} k_{P}}=$ $\hat{b}_{m^{*} k_{P}}+1$, calculate $\Delta P_{m^{*} k_{P}}$ as in (15), $\Delta P_{m k_{P}}=$ $\infty, \forall m \neq m^{*}$, and go to step 2(a).

(d) If $P_{\mathrm{SU}}+\Delta P_{m^{*} k_{P}}>P_{\text {total }}$, then set $m^{*}$ to be the user with the next higher value of $\widehat{B}_{m} / \lambda_{m}$ and go to step 2(b). Stop if all users have been considered.

Similarly, the m-MI algorithm is used to determine the total power, $P_{\mathrm{MI}}$, required for transmitting to the SUs if, at each bit loading, we choose the subchannel which minimizes the incremental interference power introduced into the PU band.

\section{Algorithm m-MI}

(1) Step 1-Initialization

(a) Set $P_{\mathrm{MI}}=0, I_{\mathrm{PU}}=0$

(b) Set $\widehat{B}_{m}=0$ for $m \in\{1,2, \ldots, M\}$.

(c) Set $\hat{b}_{m k}=0$ and calculate $\Delta I_{m k}$ as in (16), for $m \in$ $\{1,2, \ldots, M\}$ and $k \in\{1,2, \ldots, K\}$.

(2) Step 2

(a) Determine $m^{*}=\arg \min _{m} \widehat{B}_{m} / \lambda_{m}$; ties are first broken in decreasing order of $\lambda$ then randomly.

(b) Determine $k_{I}=\arg \min _{k} \Delta I_{m^{*} k}$.

(c) If $I_{\mathrm{PU}}+\Delta I_{m^{*} k_{I}} \leq B_{I} I_{\mathrm{th}}$, perform the following updates: $\widehat{B}_{m^{*}}=\widehat{B}_{m^{*}}+1, I_{\mathrm{PU}}=I_{\mathrm{PU}}+\Delta I_{m^{*} k_{I}}, P_{\mathrm{MI}}=P_{\mathrm{MI}}+$ $\Delta I_{m^{*} k_{I}} / I F_{k_{I}}, \hat{b}_{m^{*} k_{I}}=\hat{b}_{m^{*} k_{I}}+1$, calculate $\Delta I_{m^{*} k_{I}}$ as in (16), set $\Delta I_{m k_{I}}=\infty, \forall m \neq m^{*}$, and go to step 2(a).

(d) If $I_{\mathrm{PU}}+\Delta I_{m^{*} k_{I}}>B_{I} I_{\mathrm{th}}$, then set $m^{*}$ to be the user with the next higher value of $\widehat{B}_{m} / \lambda_{m}$ and go to step 2(b). Stop if all users have been considered.

The relative importance of the $\mathrm{SU}$ power and $\mathrm{PU}$ interference is measured using

$$
V P=\frac{P_{\mathrm{MI}}-P_{\text {total }}}{P_{\text {total }}}, \quad V I=\frac{I_{\mathrm{MP}}-B_{I} I_{\mathrm{th}}}{B_{\mathrm{I}} I_{\mathrm{th}}},
$$

respectively. Note that $V P$ is negative when $P_{\text {total }}>P_{\mathrm{MI}}$, and $V I$ is negative when $B_{I} I_{\mathrm{th}}>I_{\mathrm{MP}}$.

The $\mathrm{m}$-RC algorithm uses $V I$ and $V P$ as follows.

\section{Algorithm $m-R C$}

(1) Step 1-Initialization

(a) Set $P_{\mathrm{SU}}=0, I_{\mathrm{PU}}=0$

(b) Set $\widehat{B}_{m}=0$ for $m \in\{1,2, \ldots, M\}$.

(c) Set $\hat{b}_{m k}=0$, and calculate $\Delta P_{m k}$ as in (15) and $\Delta I_{m k}$ as in (16), for $m \in\{1,2, \ldots, M\}$ and $k \in\{1,2, \ldots, K\}$.

(2) Step 2

(a) Determine $m^{*}=\arg \min _{m} \widehat{B}_{m} / \lambda_{m}$; ties are first broken in decreasing order of $\lambda$ then randomly.

(b) Determine $k_{P}=\arg \min _{k} \Delta P_{m^{*} k}$.

(c) Determine $k_{I}=\arg \min _{k} \Delta I_{m^{*} k}$.

(d) Compute $X=V I\left(\Delta P_{m^{*} k_{P}} I F_{k_{P}}-\Delta I_{m^{*} k_{I}}\right) / \Delta I_{m^{*} k_{I}}$ and $Y=V P\left(\Delta I_{m^{*} k_{I}} / I F_{k_{I}}-\Delta P_{m^{*} k_{P}}\right) / \Delta P_{m^{*} k_{P}}$

(e) If $X \geq Y$, set $k^{*}=k_{I}$; otherwise set $k^{*}=k_{P}$.

(f) If $P_{\mathrm{SU}}+\Delta P_{m^{*} k^{*}} \leq P_{\text {total }}$ and $I_{\mathrm{PU}}+\Delta I_{m^{*} k^{*}} \leq$ $B_{I} I_{\text {th }}$, perform the following updates: $\widehat{B}_{m^{*}}=\widehat{B}_{m^{*}}+$ $1, \hat{b}_{m^{*} k^{*}}=\hat{b}_{m^{*} k^{*}}+1, I_{\mathrm{PU}}=I_{\mathrm{PU}}+\Delta I_{m^{*} k^{*}}, P_{\mathrm{SU}}=P_{\mathrm{SU}}+$ $\Delta P_{m^{*} k^{*}}$, calculate $\Delta P_{m^{*} k^{*}}$ as in (15) and $\Delta I_{m^{*} k^{*}}$ as in (16), set $\Delta P_{m k^{*}}=\infty, \Delta I_{m k^{*}}=\infty$, and $\forall m \neq m^{*}$, and go to step 2(a). 


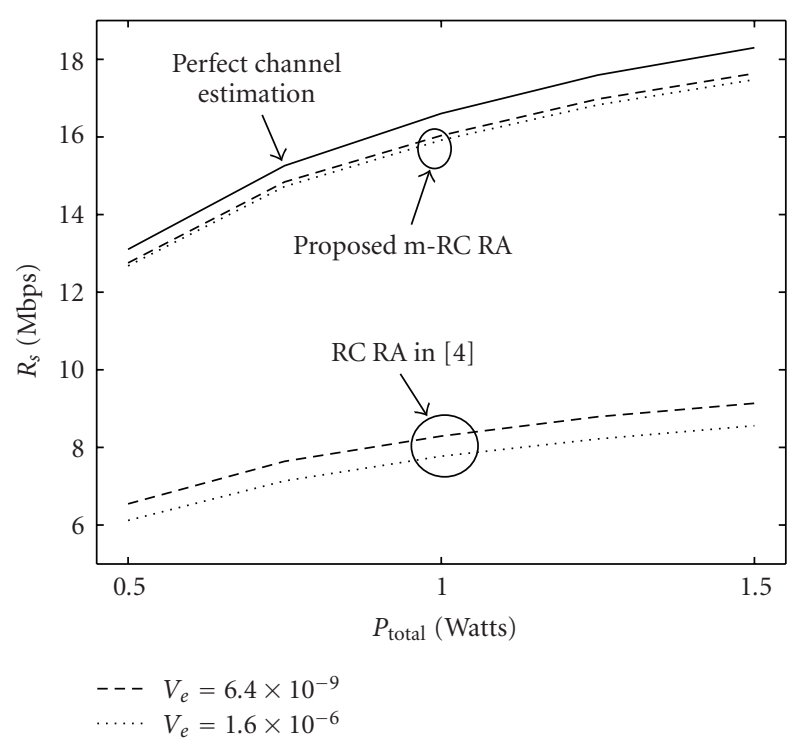

Figure 1: Total bit rate, $R_{s}$, as a function of $P_{\text {total }}$ with $I_{\text {th }}=6 \times$ $10^{-6} \mathrm{~W}$.

(g) If $P_{\mathrm{SU}}+\Delta P_{m^{*} k^{*}}>P_{\text {total }}$ or $I_{\mathrm{PU}}+\Delta I_{m^{*} k^{*}}>B_{I} I_{\mathrm{th}}$, then set $m^{*}$ to be the user with the next higher value of $\widehat{B}_{m} / \lambda_{m}$, and go to step 2(b). Stop if all users have been considered.

(h) Calculate $b_{m k}$ as in (5),

If $\hat{b}_{m k}>b_{m k}$, then set $b_{m k}=0$. Otherwise, set $b_{m k}=$ $\hat{b}_{m k}$. Update $B_{m}$.

Good values for the back-off factors, $B_{G}$ and $B_{I}$, are chosen as follows

(1) For given values of $P_{\text {total }}$ and $I_{\text {th }}$, both $P_{o}$ and the throughput, $R_{s}$, increase with $B_{I}$, whereas $B_{G}$ has little effect on $P_{o}$. We therefore choose to use the largest value of $B_{I}$ which can satisfy the $P_{o}$ requirement.

(2) Once the value of $B_{I}$ is chosen, we determine the throughput for different values of $B_{G}$ and select the $B_{G}$ value which yields the highest throughput.

\section{Results}

Computer simulations were run with the proposed m-RC RA algorithm assuming that the PU signal is an elliptically filtered white noise process and using the same parameter values as in [4]. In addition, let $V_{e}$ denote the variance of the real (or imaginary) part of the channel estimation error in (12).

Figure 1 shows the total SU bit rate, $R_{s}$, as a function of $P_{\text {total }}$ with $I_{\text {th }}=6 \times 10^{-6} \mathrm{~W}$ for two different values of $V_{e}$ and three cases: (1) perfect channel estimation, (2) channel estimation errors with the RC RA algorithm in [4], and (3) channel estimation errors with the proposed m-RC RA algorithm. It can be seen that in the presence of channel estimation errors, the RC RA algorithm in [4] shows a big

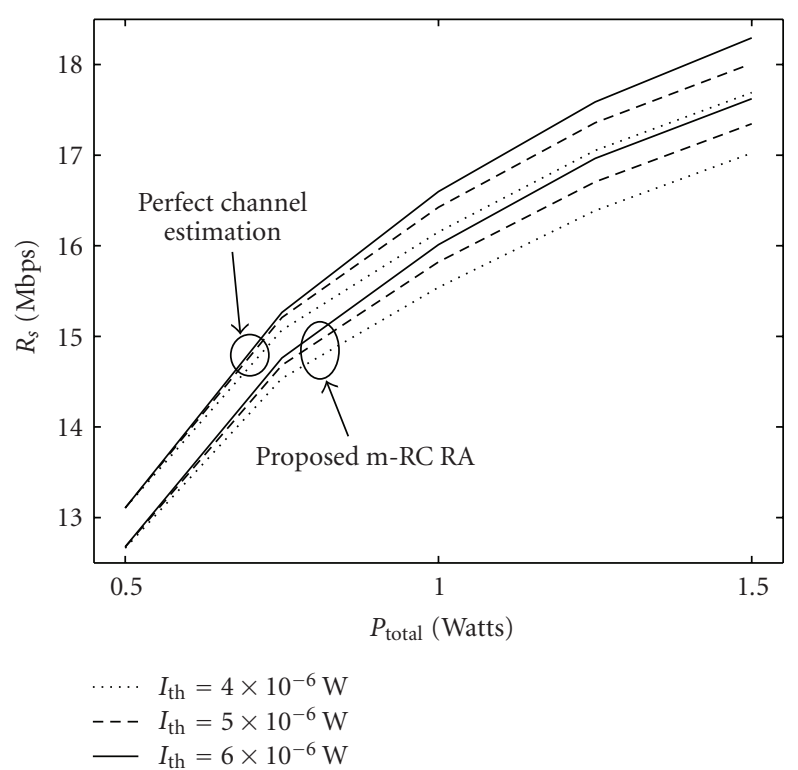

FIgURe 2: Total bit rate, $R_{s}$, as a function of $P_{\text {total }}$ for the m-RC RA algorithm with $P_{o}=10^{-3}$.

drop in $R_{s}$ compared to the perfect channel estimation case. Furthermore, $P_{o}$ for the RC RA algorithm in [4] can be as high as $6 \%$. Using the m-RC RA algorithm with $B_{G}=0.84$ and $B_{I}=0.815$, the loss relative to the perfect channel estimation case is small, and $P_{o}$ is negligibly small. The results show that the proposed $\mathrm{m}$-RC RA is quite robust against channel estimation errors.

Figure 2 shows $R_{s}$ for the m-RC RA algorithm as a function of $P_{\text {total }}$ for different values of $I_{\text {th }}$. It was found from the simulation results that a $B_{G}$ value of 0.84 is nearoptimal for maximizing $R_{s}$. In this figure, $B_{I}$ was set to 0.905 to achieve a $P_{o}$ of $10^{-3}$. As expected, $R_{s}$ increases with $I_{\text {th }}$. For comparison, the perfect channel estimation curves are also included in Figure 2. It can be observed that the $R_{s}$ value for $\mathrm{m}$-RC RA is less than 5\% lower.

\section{Conclusion}

A simple back-off scheme was proposed to counter the deleterious effect of channel estimation errors in a multiuser OFDM CR system. Simulation results show that the proposed scheme can greatly reduce the loss in the total bit rate to SUs.

\section{Acknowledgment}

This research was supported in part by the Singapore Ministry of Education Grant no. RGM24/06.

\section{References}

[1] J. Mitola III and G. Q. Maguire Jr., "Cognitive radio: making software radios more personal," IEEE Personal Communications, vol. 6, no. 4, pp. 13-18, 1999. 
[2] S. Haykin, "Cognitive radio: brain-empowered wireless communications," IEEE Journal on Selected Areas in Communications, vol. 23, no. 2, pp. 201-220, 2005.

[3] T. A. Weiss and F. K. Jondral, "Spectrum pooling: an innovative strategy for the enhancement of spectrum efficiency," IEEE Communications Magazine, vol. 42, no. 3, pp. S8-S14, 2004.

[4] T. Qin and C. Leung, "Fair adaptive resource allocation for multiuser OFDM cognitive radio systems," in Proceedings of the 2nd International Conference on Communications and Networking in China, (ChinaCom '07), pp. 115-119, August 2007.

[5] Z. Hu, G. Zhu, Y. Xia, and G. Liu, "Multiuser subcarrier and bit allocation forMIMO-OFDM systems with perfect and partial channel information," in Proceedings of the IEEE Wireless Communications and Networking Conference, vol. 2, pp. 11881193, March 2004.

[6] T. Weiss, J. Hillenbrand, A. Krohn, and F. K. Jondral, "Mutual interference in OFDM-based spectrum pooling systems," Proceedings of the IEEE Vehicular Technology Conference (VTC '04), vol. 59, no. 4, pp. 1873-1877, 2004.

[7] J. Jang and K. B. Lee, "Transmit power adaptation for multiuser OFDM systems," IEEE Journal on Selected Areas in Communications, vol. 21, no. 2, pp. 171-178, 2003. 

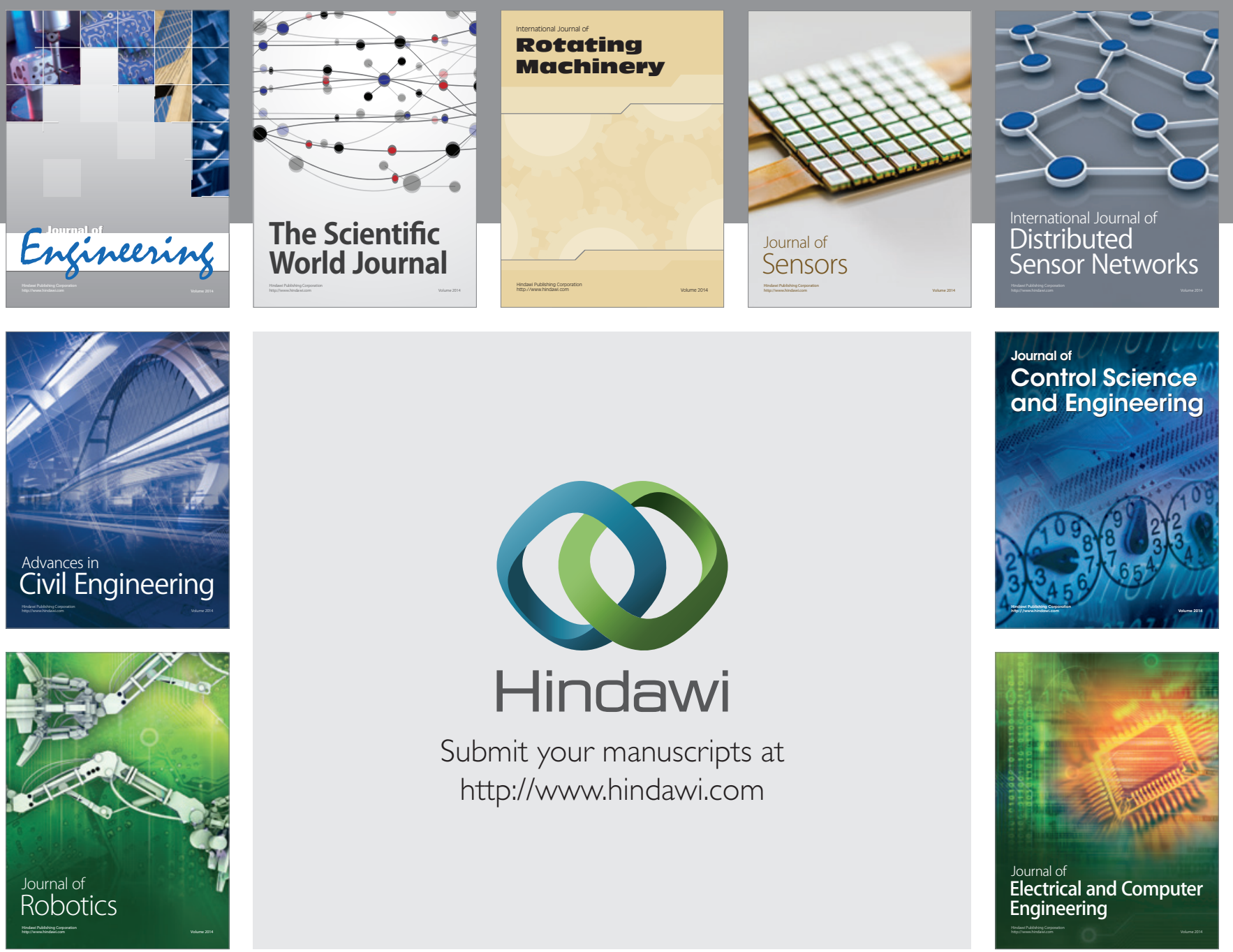

Submit your manuscripts at

http://www.hindawi.com
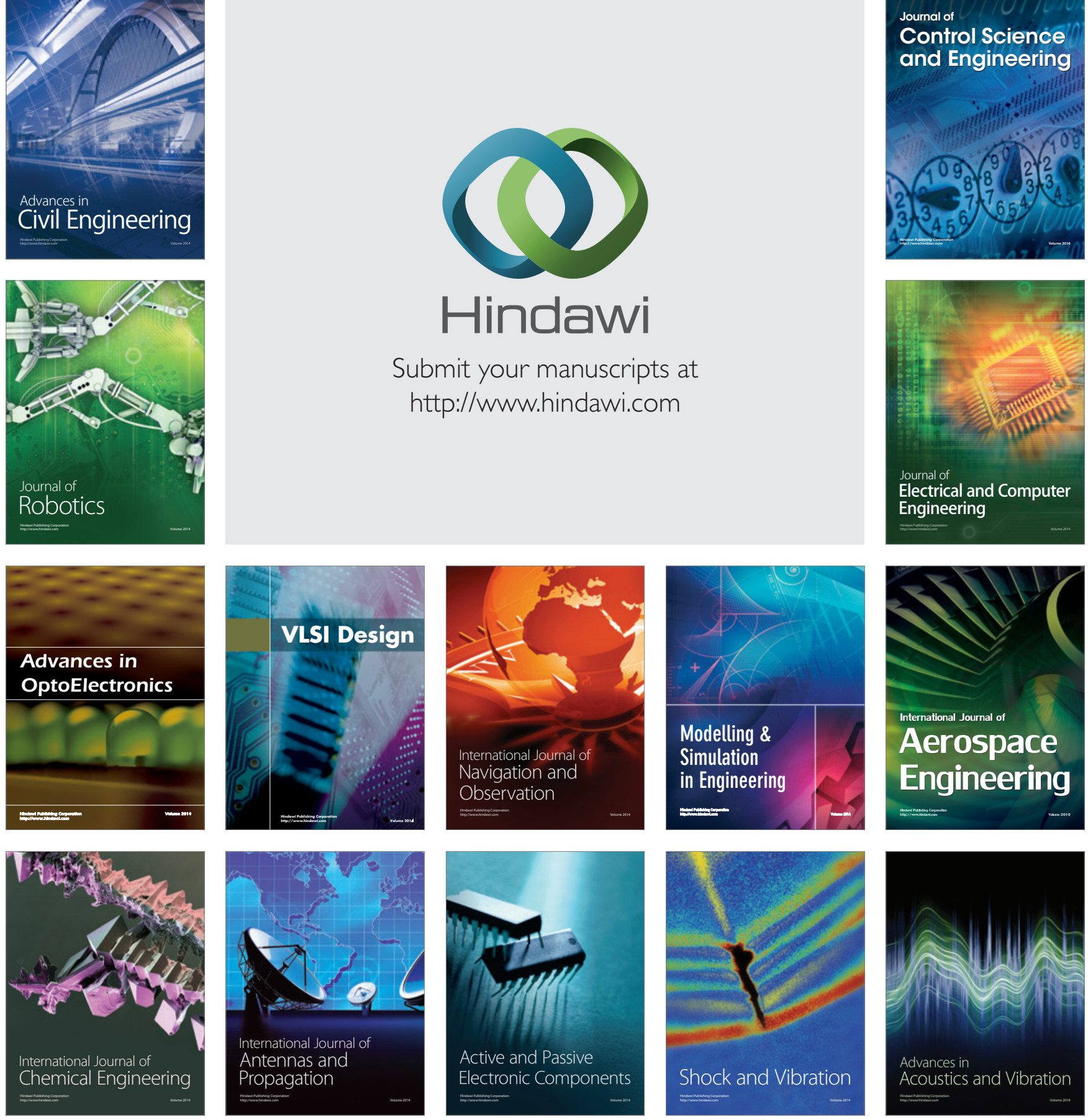